\title{
Neuropatía femoral en cirugía urológica
}

\author{
Pastor Guzmán JM, Pastor Navarro H, Donate Moreno MJ, Pardal Fernández JM*, \\ Carrión López P, Salinas Sánchez A, Virseda Rodríguez JA.
}

Servicio de Urología. Servicio de Neurofisiologí**.Hospital General Universitario. Albacete

Actas Urol Esp. 2007;31(8):885-894

\section{RESUMEN}

NEUROPATÍA FEMORAL EN CIRUGÍA UROLÓGICA

Se presentan cuatro casos de neuropatía femoral secundarios a cirugía urológica, el primero tras lumbotomía derecha hace más de 20 años y los otros tres en los últimos cuatro años, con incisión iliaca. Se comentan los mecanismos de producción de la lesión, evolución, tratamiento y prevención de esta infrecuente complicación neurológica y se revisa la literatura sobre dicha patología en la actividad urológica.

Palabras clave: Neuropatía femoral. Atrofia. Cuadriceps. Valvas del separador.

\section{ABSTRACT \\ FEMORAL NEUROPATHY IN UROLOGICAL SURGERY}

We present four cases of femoral neuropathy due to urological surgery, first case happened after right lumbotomy twenty years ago and the other three cases in the last four years after iliac incision. We review lesion production mecanism, evolution, treatment and prevention of this rare neurological complication. We do a literature review about this pathology related with urological activity.

Keywords: Femoral Neuropathy. Atrophy. Quadriceps. Retractor valves.

$\mathrm{L}$ as lesiones quirúrgicas de nervios periféricos son complicaciones relativamente frecuentes, pudiendo darse también en procedimientos urológicos presentándose como una plexopatía lumbosacra, o una mononeuropatía que es lo más común. En ocasiones pueden no ser advertidas, por la escasa significación de la sintomatología o su breve duración. La lesión nerviosa se puede provocar por traumatismo directo, isquemia nerviosa o compresión. Ocurre con mayor frecuencia en la cirugía pélvica y en posición de litotomía. Las publicaciones que refieran esta patología son escasas; aportamos cuatro casos, tres en incisión iliaca y otro en lumbotomía, producidos por la compresión ejercida por las valvas de los separadores. El pronóstico es bueno en la mayoría de los pacientes.

\section{CASOS CLÍnICOS}

\section{Caso 1}

Varón de 63 diagnosticado de litiasis piélica de riñón derecho ptósico. Se efectuó pielolitectomía mediante lumbotomía baja, por debajo de XII costilla, sin incidencia intraoperatoria alguna. La duración de la intervención fue de 90 minutos. A las 24 horas de la intervención el paciente refiere dolor en cara anterior de muslo derecho, leve dificultad para flexionar la cadera e imposibilidad de extender la rodilla. El reflejo rotuliano estaba abolido. El primer estudio electrofisiológico, a las tres semanas de la intervención, mostró lesión del nervio femoral. Se interpretó como lesión en zona alta del nervio femoral, provocada por compresión indirecta del mismo por las ramas del separador apoyadas sobre músculo psoas. A 
pesar de la rehabilitación precoz se produjo una rápida atrofia cuadricipital. La evolución fue tórpida, pues la atrofia muscular tardó en resolverse un año, pudiendo abandonar el apoyo de muletas entonces. El último control del que tenemos constancia fue cuatro años después, en el que se apreció leve disminución del perímetro del muslo derecho, respecto al izquierdo, persistiendo leve dificultad a la deambulación, aunque no precisaba de apoyos externos para la misma.

\section{Caso 2}

Mujer de 28 años, sin antecedentes de interés, ingresada tres meses antes de la intervención por dolor lumbar izquierdo y fiebre con escalofríos, siendo diagnosticada de pielonefrítis aguda izquierda. En Urografias I.V. el riñón derecho era normal; riñón izquierdo con retraso funcional, con comienzo del relleno calicial a los 30 minutos, $\mathrm{y}$ en $\mathrm{Rx}$ retardadas ureteropielocaliectasia importante de inicio a nivel de uréter iliaco sin apreciar motivo de la obstrucción. En TAC abdominopélvico solo se demostró la dilatación ureteropielocalicial, pero no objetivó patología intrínseca o extrínseca en uréter iliopélvico. Se procedió a colocación de catéter ureteral izquierdo no progresando el mismo a nivel aproximado de cruce iliaco, comprobándose en ureteropielografía retrógrada la existencia de una estenosis. Estudiada por ginecología con ecografía transvaginal, se descarta patología de dicha especialidad.

Se practicó incisión iliaca de 6-7 cm(Mc Burney izquierdo), con disección roma de la musculatura abdominal y acceso extraperitoneal, ureterolisis amplia de uréter iliopélvico y ureterectomía segmentaria de 3-4 cm con anastomosis terminoterminal. La duración de la intervención fue de 80 minutos. A las 24 horas de la intervención y con buen estado general, la paciente se levanta de la cama pero refiere que no puede deambular sin ayuda, por perdida de fuerza de la pierna izquierda. La exploración neurológica evidencia debilidad proximal de miembro inferior izquierdo, siendo imposible la extensión de la pierna, hipoestesia en cara anterior de muslo en tercio distal y abolición del reflejo rotuliano ipsilateral; indemnidad del iliopsoas.
En TAC abdominopélvico postoperatorio no se evidenciaron hematomas retroperitoneales o de la musculatura iliopsoas.

Se realizaron 3 estudios neurofisiológicos, a los 8 días de evolución, a las 3 y a las 8 semanas. Se estudiaron los componentes sensitivo (safeno) y motor (femoral) del nervio, así como otros territorios que pudieran plantear diagnóstico diferencial (musculatura miotómica de raíz L3 y L4 no femoral, plexo lumbar, polineuropatía, etc.). En todas las exploraciones se evidenciaron criterios muy predominantes de desmielinización nerviosa del nervio femoral, con muy escasos datos de lesión axonal asociada. A la $8^{\mathrm{a}}$ semana de evolución fue objetivable una recuperación funcional de la musculatura afectada prácticamente ad integrum.

El tratamiento que se prescribió incluyó rehabilitación precoz, que continuó hasta la resolución del proceso. Durante las primeras tres semanas se administraron vitaminas del complejo B y corticoterapia. A las 8 semanas de la intervención comienzan a mejorar los trastornos sensitivos y una semana después recupera la movilidad de la musculatura afectada progresiva $\mathrm{y}$ rápidamente. A los cuatro meses la sensibilidad y movilidad de la pierna eran normales, aunque continuó con ejercicios de potenciación muscular por persistir moderada atrofia del cuadriceps.

\section{Caso 3}

Varón de 63 años con insuficiencia renal crónica de origen no filiado, probablemente por nefropatía intersticial. Recibió transplante renal de donante cadáver, en fosa iliaca izquierda; la duración de la intervención fue de 1 hora y 50, tiempo de isquemia caliente de 55 minutos, con diuresis inmediata y función renal normal el duodécimo día postoperatorio en que fue dado de alta. Al día siguiente de la intervención nota disminución de la fuerza en pierna izquierda, sufriendo una caída al inicio de la deambulación; al alta hospitalaria había mejorado algo la potencia y movilidad de la pierna. A la exploración neurológica se comprobó la ausencia de reflejo patelar, la disminución de potencia del cuadriceps y los trastornos de sensibilidad en cara anterior de muslo. El estudio neurofisiológico a los 10 días de evolución sugería neuroapraxia del nervio 
femoral. Asímismo se efectuó estudio del reflejo patelar o rotuliano apreciando aumento significativo de la latencia del reflejo, expresión inequívoca de componente desmielinizante, no siendo descartable la lesión axonal asociada; en un segundo estudio realizado varias semanas después se recupera la latencia aunque persiste amplitud pequeña, en probable relación con bloqueo parcial de la conducción (desmielinización parcial del nervio) (Fig 1). No aceptó inmediatamente el tratamiento rehabilitador, comenzando éste 7 semanas después al persistir el trastorno neurológico, existiendo ya evidente atrofia de muslo izquierdo; fue tratado con ejercicios isométricos de cuadriceps y galvanización. A los seis meses recuperó la potencia muscular, desapareciendo los trastornos de la sensibilidad ; a la exploración física el reflejo patelar izquierdo dos años después de la intervención es menos vivo que el derecho.

\section{Caso 4}

Paciente de 44 años con antecedentes de: insuficiencia renal secundaria a poliquistosis hepatorrenal; hipotiroidismo; nefrectomía derecha para dejar libre fosa iliaca para futuro transplante. Se efectuó transplante renal de donante cadáver en fosa iliaca derecha, con tiempo operatorio de 2 horas, e isquemia caliente de 52 minutos, con inicio inmediato de diuresis descubriéndose a las 12 horas de la intervención monoparesia de miembro inferior derecho, apreciando la exploración neurológica: paresia exclusiva del cuadriceps derecho, hipoestesia de la cara anteromedial del muslo y de la pierna, con abolición del reflejo patelar. El estudio mediante TAC abdominal, a las 24 horas del transplante fue normal y sin hematomas del área quirúrgica. Se produjo necrosis tubular que requirió diálisis hasta el décimo día postoperatorio, en que rápidamente mejoró la función renal, siendo alta hospitalaria diez días después con función renal normal.

Se efectuó EMG, al $10^{\circ}$ día, informándose como neuropatía femoral derecha de intensidad severa, con las características de una afectación desmielinizante, aunque aun no era descartable una lesión axonal asociada. Se repitió el estudio neurofisiológico 10 días después, comprobando la mejoría de la neuropatía, persistiendo los datos que sugieren buen pronostico funcional. El estudio neurofisiológico del reflejo patelar al décimo día mostró un patrón desmielinizante $\mathrm{y}$ varias semanas después la normalización del mismo (Fig 2). Inició rehabilitación precoz comenzando la misma al $4^{\circ}$ día de la intervención. A su alta hospitalaria, a las tres semanas del transplante había mejorado llamativamente la movilidad de la pierna, con deambulación fácil con la ayuda de un bastón, aunque todavía no extendía completamente la pierna y el reflejo rotuliano seguía estando abolido. A los cinco meses de la intervención, la movilidad, el reflejo rotuliano y la potencia muscular del miembro afecto eran normales. $2^{\circ}$ estudio, varias semanas después. Normalización de latencia. persiste bloqueo (desmielinización). 
En tres pacientes se efectuó ELECTRONEUROGRAFÍA SENSITIVA (ENG), con estudios en diferentes fases evolutivas demostrando una completa normalidad del nervio safeno del lado sintomático. Este parámetro indica la ausencia de lesión axonal (degeneración Walleriana) de importancia, lo que sumado a la información del reflejo antes descrito confirmaba el buen pronóstico de la lesión femoral (desmielinización). En la Figura 3 están representadas las ENG de los casos 3 y 4 a los 10 días de producida la lesión.

\section{DISCUSIÓN}

El plexo lumbar esta formado por una rama ventral que va de T12 a los nervios espinales de L4, que se unen en la masa del músculo psoas mayor. Los nervios mayores que se incluyen en este plexo son: femoral, obturador, ilioinguinal, iliohipogástrico, genitofemoral y cutáneo femoral lateral. Inervan los músculos cuya acción es: flexión de la cadera, extensión de la rodilla, adducción de la pierna; y proporciona sensibilidad a: zona inferior de hipogastrio, parte medial de la pierna, escroto en el varón y labios mayores en la mujer. Las lesiones de plexo parecen ser esporádicas en comparación con la mononeuropatía ${ }^{1}$ femoral, y esta es relativamente infrecuente comparada con otras mononeuropatías ${ }^{2,3}$.

El nervio femoral es la rama principal originada en el plexo lumbar, formándose por la unión de las ramas ventrales de las divisiones posteriores de las raíces $\mathrm{L} 2, \mathrm{~L} 3, \mathrm{~L} 4$ (Fig. 4) .

Desciende oblicuamente dentro del músculo psoas mayor, emergiendo desde la parte inferolateral del mismo, por el surco formado entre el psoas y el iliaco, dando ramas motoras a ambos músculos. Cursa después posterolateralmente a los vasos iliacos, para descender al muslo a través del canal femoral, por debajo del ligamento inguinal (Fig. 5).

En esa zona, a 1-1,5 cm distal al ligamento, se divide en sus ramas terminales motoras que inervan al cuadriceps femo-

FIGURA 3. Electroneurografia sensitiva del nervio safeno, casos 3 y 4 . 


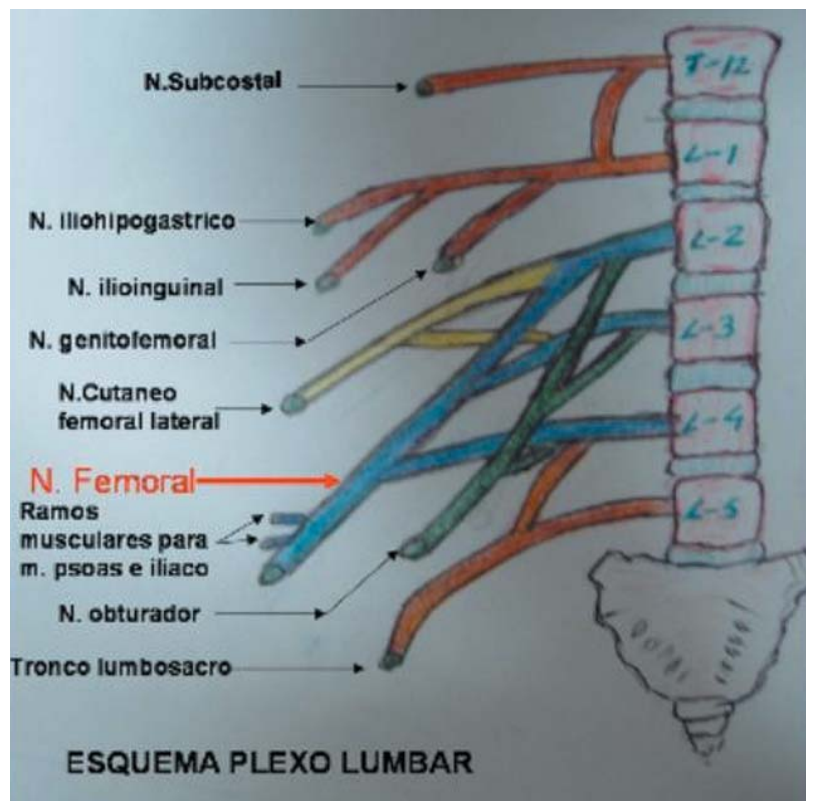

FIGURA 4. Esquema plexo lumbar

ral, pectíneo y sartorio, y sensitivas que inervan la cara anteromedial del muslo y pierna, mediante los nervios femorocutáneo anterior, femorocutáneo medial y safeno largo ${ }^{4-8}$.

Las lesiones del plexo lumbar en general, y mas específicamente del nervio femoral, se pueden producir por múltiples causas: en enfermedades sistémicas y metabólicas como la diabetes, sífilis, TBC, poliarteritis nodosa, uremia y alcoholismo; por hematomas retroperitoneales espontáneos o de los músculos iliaco

o psoas (como en discrasias sanguíneas o en tratamientos anticoagulantes); por traumatismos con heridas abiertas, fracturas de cadera y pelvis; enfermedades del triángulo femoral; en patología oncológica como linfomas, metástasis y tumores retroperitoneales; infecciones que producen abscesos iliacos o del psoas; aneurismas aortoiliacos; en embarazo y parto; en gimnastas por hiperextensión de la cadera; en procedimientos de cateterización de arteria femoral, bloqueo anestésico de nervios ilioinguinales y radioterapia. Pero un mecanismo lesional del nervio femoral muy peculiar, es el producido en intervenciones quirúrgicas, como en las de cadera, pero más frecuentemente en cirugias abdominales y mayoritariamente pélvicas.

Las lesiones intraoperatorias de nervios periféricos de miembros inferiores son complicaciones relativamente frecuentes, aunque se descubran horas, días o semanas después de la cirugía. Parece que su número es mucho mayor de lo publicado, unas veces porque no se reconoce, al producir una patología o sintomatología de escasa entidad y muy transitoria, y otras se confunde con alteraciones o molestias que se dan como habituales en los postoperatorios, como puede ocurrir en casos leves donde únicamente hay síntomas sensitivos como el adormecimiento. Pueden presentarse como mononeuropatía o con lesiones extensas y mixtas del plexo lumbosa$\mathrm{cro}^{1}$. Si se produce afectación del nervio femoral y cuando el déficit, sobre todo motor, es importante, la sospecha clínica se hace muy evidente, comportando severa incapacidad para el paciente y motivo de preocupación para el cirujano.

La primera descripción de la neuropatía femoral aislada se debe a Descot en 1822 como "anterior crural neuritis"9,10. En 1896 es publicado el primer caso de neuropatía femoral como complicación de histerectomía abdominal ${ }^{11}$. Garrigues

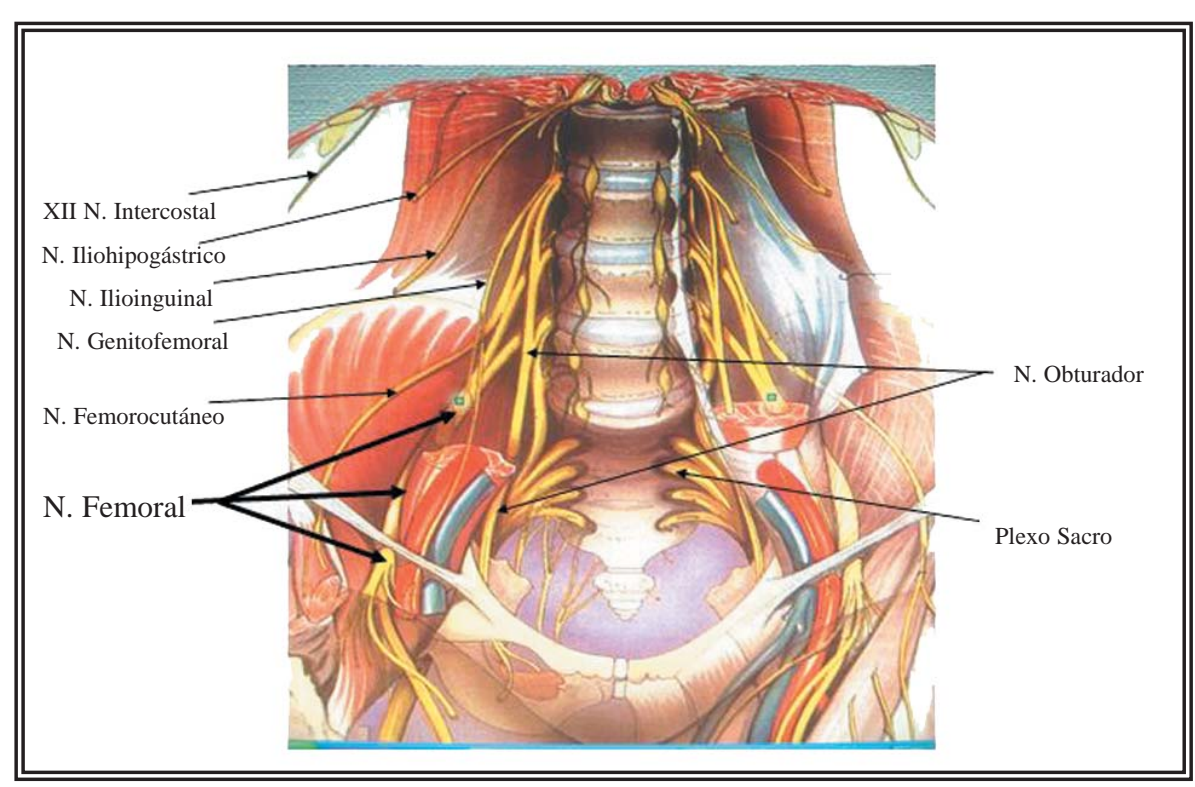

FIGURA 5. Plexo lumbosacro. 
en 1897 comunica el primer caso de neuropatía femoral en histerectomía vaginal ${ }^{9}$. Desde entonces se han comunicado multitud de casos pero la incidencia es incierta.

Las causas se pueden dividir en tres categorías ${ }^{5}$ : a) Traumatismo directo del nervio por cauterización, sección o sutura. b) Isquemia nerviosa por interrupción de la vascularización a nivel intrapélvico, como puede ocurrir en la cirugía vascular, de aorta o en el transplante renal. c) Por compresión directa o indirecta del nervio por hematomas o por las valvas de los separadores. En la producción de la lesión a veces se asocia mas de un mecanismo de los descritos, como sucede en las lesiones provocadas por los separadores, que es la más frecuente, existiendo compresión y por ello también interrupción de la vascularización, edema, posible ruptura capilar y secundariamente hematoma y fibrosis perineu$\mathrm{ral}^{12,13}$. Casi siempre se trata de lesiones nerviosas en continuidad.

La incidencia estimada ${ }^{6,14}$ en la cirugía abdominal y como consecuencia de la compresión del nervio por las valvas de los separadores es de $0,12 \%$.

Aunque de presentación infrecuente se ha descrito en cirugía digestiva(cirugia de colon y recto $^{14,15}$, herniorrafias, apendicectomías ${ }^{6}$. .etc...)

En cirugía aortoiliaca ${ }^{16}$, por arterioesclerosis o aneurismas, donde se ocluye temporalmente la aorta o las arterias iliacas.

Se argumenta que es más frecuente en la cirugía pélvica con incisiones bajas transversales ${ }^{3,17}$, como en la transrectal de Mouchel o en la de Pfannenstiel; ambas y sobre todo la segunda ampliamente utilizadas en la cirugía ginecológica. Por eso quizás el mayor número de casos publicados sea en la cirugía de esta especialidad. En estudio prospectivo ${ }^{18}$ de 1981 en histerectomías abdominales, se comparan el tipo de incisión quirúrgica, el tiempo quirúrgico, la experiencia de varios cirujanos y el uso de tres separadores autostáticos distintos, comprobando la alta incidencia de neuropatía femoral(11,6\%), concluyendo que en la aparición de esta patología no es estadísticamente significativo ni la superación del tiempo medio quirúrgico, ni el tipo de incisión, ni la experiencia del cirujano, ni tampoco el tipo de separador, pero si la longitud de las val- vas laterales, sospechando que los casos con escasa sintomatología no son bien reconocidos en el postoperatorio por lo que la incidencia en la literatura es mucho menor. En otro estudio prospectivo $^{8}$ de 1985 con dos grupos, en el primero con casi 4000 cirugías pélvicas, y el segundo con cerca de 3000 , la mayoría histerectomías, utilizando autoretractores en el primero, y solo valvas flexibles de mano en el segundo, comprueba que se produce neuropatía femoral en el $7,45 \%$ en aquel y el $0,7 \%$ en este, y concluye que al eliminar la compresión continua que ejercen los separadores autostáticos, se evita el riesgo de provocar la neuropatía.

En traumatología se produce en la cirugía de la cadera: artroplastia total, desplazamiento de prótesis, extrusión del cemento utilizado en estas cirugias, hematomas, cirugias iterativas incluidas las artroscopia y fibrosis intensas con el encapsulamiento o estrechamiento nervioso ${ }^{1,6}$.

La disfunción nerviosa puede producir en la extremidad afecta parestesia, anestesia, dolor y paresia. La clínica suele iniciarse al día siguiente de la intervención, cuando el paciente trata de levantarse y caminar y tiene dificultades o no puede hacerlo por la debilidad de los músculos extensores de la pierna (cuadriceps femoral) y flexores de la cadera (iliopsoas); puede existir inestabilidad de la rodilla e imposibilidad de elevar la pierna extendida. Se asocia a sensación de adormecimiento, parestesias o más raramente hiperestesia en la zona anteromedial de todo el miembro inferior ${ }^{4,5,7}$. El signo patognomónico es la disminución o abolición del reflejo rotuliano.

El nervio puede ser comprimido a cualquier nivel de su recorrido, pero es particularmente susceptible dentro del cuerpo del psoas, en el surco iliopsoas y en el ligamento inguinal.

La mayoría de las lesiones son unilaterales, aunque se ha descrito algún caso con afectación bilateral $^{7,19,20}$. y el mecanismo mas aceptado es la isquemia directa de la vasa nervorum. La zona intrapélvica mas vulnerable parece ser 4-6 cm por encima del ligamento inguinal, cuando empieza a salir de la musculatura psoica, pues existe deficiente vascularización ya que ese segmento nervioso solo recibe una pequeña rama de la arteria iliolumbar, rama a su vez de la iliaca interna, y otra de la arteria circunfleja profunda, 
rama de la iliaca externa, aunque en el lado derecho existe un aporte de irrigación complementaria, pues esta última arteria da mas ramas al nervio y existen anastomosis con la $4^{\mathrm{a}}$ y $5^{\mathrm{a}}$ arterias lumbares ${ }^{1,16}$. El nervio femoral izquierdo es mas frecuentemente lesionado al parecer porque tiene menos irrigación que el derecho. Fuera de la pelvis, en el muslo, la vascularización del nervio se debe a las arterias circumflejas lateral y media, ramas de la arteria iliaca externa, más generosa también en lado derecho ${ }^{4,5,7,12,16}$.

El mecanismo que provoca la lesión neurológica en las intervenciones quirúrgicas puede ser por traumatismo directo con sección del mismo, que es excepcional, por ligadura y por compresión. El mecanismo más habitual es la compresión del nervio, por las valvas de los retractores o separadores, la mayoría de las veces se trata de una compresión indirecta, ejercida sobre el músculo psoas, al estar apoyadas las valvas en el mismo, y producirse la compresión del nervio entre aquel y la pared pélvica.

El nervio en la cirugía pélvica no es visible en el campo quirúrgico, solo en $3-4 \mathrm{~cm}$ proximal al ligamento inguinal no esta protegido y oculto por el músculo psoas, aquí está cubierto por la fascia iliaca, transcurriendo entre el borde externo del psoas y el músculo iliaco (Fig 5); las maniobras quirúrgicas se desarrollan habitualmente por encima del psoas y relativamente distantes al recorrido del nervio, por lo tanto la lesión directa es improbable ${ }^{21}$, por esto no se produce una sección (axonotmesis o neurotmesis) sino neuroapraxia (por compresión), solo se afecta la mielina, pero los axones estarán integros, produciéndose un bloqueo de transmisión, que conllevará una parálisis del cuadriceps, abolición del reflejo rotuliano e hipoestesia en la cara anterior del muslo. Los pacientes delgados, con musculatura abdominal débil y pelvis estrecha son más proclives a sufrir esta patología.

En la literatura urológica la neuropatía femoral como complicación de los procedimientos o cirugía propios de la especialidad ha recibido poca atención. Ha sido descrito en diversos procedimientos quirúrgicos, sobre todo en cirugia mayor y en ciertas posiciones en que es colocado el paciente para determinadas intervenciones o pruebas diagnosticas. Así la posición de litotomía ha sido asociada a neuropatía femoral por compresión y/o estiramiento del nervio en su trayecto por debajo del ligamento inguinal ${ }^{2,22}$, pues la rotación externa, abducción excesiva y la flexión de muslos y piernas por un tiempo prolongado, provocan isquemia en ese segmento nervioso, dotado de menor vascularización. A pesar de que esta posición es muy utilizada en las exploraciones e intervenciones urológicas no hay muchos casos publicados, la mayoría se dan en histerectomías vaginales, no obstante hay descritos algunos casos como en $\mathrm{RTU}^{23}$, y prostatectomía perineal $^{24}$.

La mayoría de los casos publicados son referidos con mayor frecuencia en cirugía pélvica mayor, como en prostatectomía radical ${ }^{25}$ o en cistoprostatectomía ${ }^{7,19,20}$, pero quizás sea de mayor importancia el tiempo de compresión del nervio que el tipo de cirugía, aunque lógicamente el tiempo empleado suele ser más prolongado en cirugía mayor.

En el transplante renal con injerto en fosa iliaca, pueden producirse lesiones nerviosas, la más frecuente es la del femorocutáneo lateral ${ }^{26}$, que es exclusivamente sensorial, por lo que en muchos casos puede pasar desapercibida; pero la del nervio femoral es mas invalidante y se refiere en alguna serie relativamente amplia ${ }^{27}, \mathrm{y}$ en algún estudio prospectivo ${ }^{21}$ que se produce neuropatía femoral entre el 0,5-2,2\% de los casos, y el mecanismo de lesión que se arguye es el mismo que en otros procedimientos urológicos, la compresión del nervio, al que se suman posibles hematomas en el espacio iliaco, y la prolongación del tiempo de anastomosis vascular o clampaje arterial; a mayor tiempo de isquemia más posibilidades de provocar neuropatía. La precocidad en la recuperación de la neuropatía en estos casos, parece depender de la buena función del injerto $^{28,29}$, aunque a otros autores les parece independiente de ésta ${ }^{26}$.

Se produjo neuropatía femoral en un caso de nefrolitotomía percutánea en riñón pélvico ${ }^{30}$, con acceso posterior y próximo a espina iliaca superior, probablemente por mecanismo doble, por hematoma en el psoas y lesión directa de las ramas del plexo lumbar antes de su reunión para constituir el nervio femoral. 
Existe un caso de neuropatía femoral provocada por hematoma retroperitoneal y del músculo iliopsoas originada en paciente con cáncer renal metastásico ${ }^{31}$.

Como en nuestro segundo caso, existe algún otro de neuropatía femoral, en cirugía del uréter iliopélvico, abordado mediante incisión iliaca ${ }^{32}$.

Se ha publicado tres casos de neuropatía femoral en la confección de vejiga psoica, atribuido a englobamiento del nervio en la sutura de fijación vesico-psoica, demostrada al menos en un caso al ser reintervenida la paciente ${ }^{33}$, retirándose un punto de sutura de polipropileno con desaparición del trastorno neurológico.

También pudieran producirse lesiones del nervio femoral en linfadenectomías ilioinguinales, ya que dicho nervio y sus ramas están muy próximas al área de disección, pues transcurre en la pelvis y en el triangulo femoral, lateral a la arteria iliaca externa y femoral respectivamente, por lo que se podría producir lesión directa del mismo(cauterización, ligadura o sección). Sin embargo no hemos encontrado publicadas complicaciones neurológicas del nervio femoral en este tipo de procedimiento quirúrgico, relativamente frecuente en patología tumoral del pene, y eso que en el borde interno del sartorio a veces se seccionan ramas cutáneas, que no acarrean problemas, pero las musculares si podrían crearlos. Solo existe una referencia ${ }^{34}$ de neuropatía femoral, por lesión directa del nervio en linfadenectomía inguinal, tras vulvectomía radical en carcinoma vaginal.

En pocos casos se ha publicado neuropatía femoral en linfadenectomías retroperitoneales ${ }^{20,35}$ y atribuidas también a la compresión por las valvas.

De nuestros cuatro casos, uno se produjo en incisión de lumbotomía y los otros tres en incisiones iliacas, todos por patologias no malignas (una litiasis, una estenosis ureteral y dos transplantes renales), sin excesivo tiempo quirúrgico, a pesar de lo cual también se produjo la neuropatía.

Consideramos que la lesión del nervio femoral secundaria a cirugia, no está condicionada por el tipo de cirugía, sea esta mayor o menor, ni siquiera por el tipo de incisión, horizontal, media o lateral, sino por la compresión de las valvas laterales del separador sobre el músculo psoas, agravada por el mayor tiempo quirúrgico, y quizás también esté en relación directa con la magnitud de la presión o tensión ejercida por dichas valvas que a veces es mayor al tratar de hacer incisiones muy económicas. Quizás en las incisiones transversales o laterales las valvas se ubican más laterales y aumentaría el riesgo.

Tampoco el tipo de separador es lo que puede condicionar esta patología y si la excesiva longitud de las valvas laterales, que con mas posibilidades e inadvertidamente se apoyan y comprimen el psoas.

El diagnóstico es clínico y la confirmación requiere estudios electrofisiológicos en los que se incluye EMG y electroneurografia (ENG). A partir del $7^{\circ}-8^{\circ}$ día de evolución es posible encontrar hallazgos que indiquen axonotmesis en ENG, en especial caídas significativas de la amplitud en los potenciales evocados sensitivos y/o motores. La confirmación absoluta se debe demorar hasta el día 15-21 de evolución, cuando podamos demostrar signos "denervativos" en la EMG del músculo afectado. Es importante demostrar la presencia de axonotmesis, porque se considera es el factor pronóstico más determinante en la evolución de una mononeuropatía. Su presencia implica una recuperación más lenta y en ocasiones incompleta, mientras que una desmielinización focal suele asociar un pronóstico magnífico en su evolución, siempre que haya desaparecido el mecanismo causal. El estudio del reflejo cuadricipital o rotuliano en nuestra experiencia aporta información muy interesante. Como cualquier reflejo de estiramiento presenta una aferencia sensitiva desde el órgano sensorial muscular cuadricipital a través del nervio femoral hasta llegar al soma de su neurona sensorial en el ganglio raquídeo vertebral, en niveles L3-L4 ipsilaterales. Sigue su camino por el cuerno posterior medular para sinaptar con la motoneurona segmentaria del asta anterior que vía también nervio femoral, provocará la contracción cuadricipital o "twitch". En ocasiones esta contracción para el ojo humano o es pequeña o incluso imperceptible, en especial en situaciones de patología, pero disponemos de tecnología para registrar incluso señales minúsculas subclínicas. Metodológicamente utilizamos un martillo de reflejos integrado y sincro- 
nizado con el electromiógrafo, registrando simultáneamente en el músculo la respuesta refleja provocada por el golpe en el tendón rotuliano con el martillo. En estos pacientes podemos encontrarnos varias posibilidades: 1) Respuesta retrasada en el tiempo, es decir, con latencia prolongada, y con diferencia notoria con respecto al contralateral, lo que sugiere lesión desmielinizante, al menos en parte, pero que permite afirmar un pronóstico razonablemente bueno. 2) Ausencia de respuesta. Al no registrar respuesta existen 2 posibilidades, o bien una desmielinización muy severa por bloqueo completo del nervio (buen pronóstico), o una axonotmesis igualmente severa (pronóstico cuando menos reservado). En esta $2^{\underline{a}}$ opción la normalidad del nervio sensitivo femoral (safeno) estudiado después de 1 semana de la lesión mediante electroneurografía convencional descartaría una Axonotmesis femoral significativa, por cuanto la presencia de daño axonal, es decir, degeneración Walleriana, haría desaparecer funcionalmente al nervio en menos de 7-8 días. Aún con estos datos es obligado el seguimiento convencional mediante EMG de musculatura cuadricipital por la presencia frecuente de cierto componente axonal asociado (secundario a desmielinización severa), habitualmente de escasa repercusión funcional en la vida diaria de los pacientes.

En nuestros pacientes la presencia de datos de desmielinización en el reflejo patelar, la normalidad de la ENG sensitiva de safeno, y la ausencia de hallazgos denervativos evidentes en la EMG, permitió emitir un pronóstico favorable en cuanto a su lesión neurológica en los primeros 8-10 días de evolución desde la cirugía.

Además del mecanismo compresivo por los retractores señalado como el mas frecuente, hay que descartar hematomas postoperatorios retroperitoneales o del iliopsoas.

El pronóstico es bueno en la mayoría de los casos. Un inequívoco pronóstico solamente es posible después de estudio EMG hecho tres o más semanas después de producida la lesión. Entre una semana y seis meses ${ }^{4,5}$ suele resolverse sin secuelas, por eso hay que tranquilizar y advertir al paciente que la incapacidad suele ser transitoria. En un estudio retrospectivo de 44 $\operatorname{casos}^{32}$ en la primera semana se curaron el
$25 \%$,en el primer mes otro $25 \%$,entre el segundo y quinto mes el $35 \%$ y el resto en el transcurso del año. Solo en un número exiguo de pacientes puede persistir después de estos plazos alguna alteración leve motora , o sobre todo de la sensibilidad $^{12}$.

El tratamiento está condicionado en base al pronóstico benigno de esta patología, provocada por compresión, que da una lesión con continuidad nerviosa con la probable recuperación espontánea. Consistirá en la rehabilitación mediante fisioterapia inmediata, para prevenir atrofia muscular, pues sino se produce ésta precozmente, y evitar las complicaciones propias de la inmovilización, especialmente las tromboembólicas. Si existe dolor se administraran analgésicos no narcóticos, a los que se puede asociar carbamacepina y/o amitriptilina ambos eficaces en el dolor neuropático. Solo en casos excepcionales será necesario recurrir a exploración quirúrgica del nervio; algunos autores ${ }^{6,12}$ recomiendan que si no hay datos clínicos o signos eléctricos de recuperación después del tercer o cuarto mes, se debe optar por la exploración quirúrgica, provocando potenciales de acción(NAP) intraoperatorios, efectuando neurolisis si se transmiten a través de la lesión, como ocurre en la mayoría de las lesiones por compresión, o resección e injerto nervioso si no es así.

En cuanto a las medidas de prevención hay que afirmar que lo mas importante es conocer la existencia de esta lesión neurológica en la actividad quirúrgica urológica y en otros tipos de cirugia, y su etiología, actuando en consecuencia; esta lesión neurológica es evitable, para lo cual se recomiendan algunas medidas sencillas como la protección con paños de la zona de apoyo de las valvas, utilizar valvas de longitud suficiente pero adecuada que solo separen la musculatura de la pared abdominal anterior(no mejora la visión del campo quirúrgico las valvas excesivamente largas si se apoyan en la musculatura de pared abdominal posterior) y comprobar que no están apoyadas en el psoas ni en los vasos iliacos, por simple inspección o introduciendo los dedos por debajo del extremo de la valva y el borde externo del psoas, verificando que hay espacio libre y suficiente entre los dos; y si la intervención es de larga duración aflojar y reposicionar regularmente el 
separador. En los procedimientos o intervenciones quirúrgicas que requieran posición de litotomía habrá que limitar la flexión, la rotación externa y la abducción de la cadera a lo estrictamente imprescindible y por el menor tiempo posible.

Nota: La Figura 5 ha sido tomada, -y modificada- de Neurología, Anatomía del sistema nervioso, Medciclopedia, www.iqb.es

\section{REFERENCIAS}

1. Collado Corona MA, Shkurovich Bialik P, Collado Ortiz Ma, Shkurovich Zaslavsky M. Lesiones perioperatorias en nervios periféricos de extremidades inferiores. Cir Ciruj 2003; 71(4):329-334.

2. Al Hakim M, Katiriji B. Femoral mononeuropathy induced by the lithotomy position: a report of 5 cases with a review of literature. Muscle Nerve 1993;16(9):891-895.

3. Rosenblum J, Schwartz G, Bendler E. Femoral neuropathy-a neurological complication of hysterectomy. JAMA 1966;195(6): 115-120.

4. Chang Canter-Colgado, Herman Montvelisky. Neuropatía femoral posthisterectomía abdominal: informe de dos casos. Rev Costarric Cienc Méd 1997;18(2):53-56.

5. Walsh C, Walsh A. Postoperative femoral neuropathy. Surg Gynecol Obstet 1992;174:255-263.

6. Kim DH, Murovic JA, Tiel RL, Kline DG. Intrapelvic and thigh-level femoral nerve lesions: management and outcomes in 119 surgically treated cases. J Neurosurg. 2004; 100(6):989-996.

7. Burnett AL, Brendler CB. Femoral neuropathy following major pelvic surgery: etiology and prevention. J Urol 1994; 151:163-165.

8. Goldman JA, Feldberg D, Dicker D, Samuel N, Dekel A. Femoral neuropathy subsequent to abdominal hysterectomy. A comparative study. Eur J Obstet Gynec Reprod Biol 1985;20:385-392.

9. Byrnes CM. Anterior crural neuritis. J Nerv Ment Dis 1913; 40: 758-78.

10. Byrnes CM. Anterior crural neuritis. J Nerv Ment Dis 1914; 41:19-31.

11. Gumpertz K. Narkoselahmung des nervos cruralis. Dtsch Med Wochenschr 1896; 22:504-505.

12. Ducic I, Dellon L, Larson EE. Treatment concepts for idiopatic and iatrogenic Femoral nerve mononeuropathy. Annals of Plastic Surgery 2005; 55(4):397-401.

13. Azuelos A, Coro L, Alexandre A. Femoral nerve entrapment. Acta Neurochir Suppl 2005; 92:61-62.

14. Dilavou ED, Anderson LR, Bernert Ra, Mularski Ra, Hunter Gc, Fiser Sm, Rappaport Wd. Lower extremity iatrogenic nerve injury due to compression during intraabdominal surgery. Am J Surg 1997; 173:504-508.

15. Celbrezze JP, Pidala MJ. Femoral neuropathy: an infrequently reported postoperative complication. Report of four cases. Dis Colon Rectum 2000; 43(3): 419-422.

16. Boontje AH, Haaxma R. Femoral neuropathy as a complication of aortic surgery. J J Cardiovasc Surg (Torino). 1987; 28:286-289.

17. Raiga J, Barakat P, Diemunsch P, Maillot C, Treisser A, Brettes JP. Paralysie du nerf fémoral dans les suites des laparotomies transversales sus-pubiennes: étude anatomi- que et hypothéses étiopathogéniques. J Gynecol Obstet Biol Reprod (Paris). 2002;31(2):183-186.

18. Kvist-Poulsen H, Borel J. Iatrogenic femoral neuropathy subsequent to abdominal hysterectomy: incidence and prevention. Obstet Gynecol 1982;60(4):516-520.

19. Corbu C, Campodonico F. Femoral nerve palsy caused by a self-retaining polyretractor during mayor pelvic surgery. Urol Int 2002; 68(1):66-68.

20. Hall MC, Koch MO. Femoral neuropathy complicating urologic abdominopelvic procedures. Urology 1995; 45(1):146149.

21. Sharma KR, Cross J, Santiago F, Ayyar DR, Burke G. Incidence of acute femoral neuropathy following renal transplantation. Arch Neurol 2002; 59(4):541-545.

22. Gombar KK, Gombar S, Singh B, Sangwan SS, Siwach RC. Femoral neuropathy: A complication of the lithotomy position. Reg Anesth 1992; 17(5):306-308.

23. Arango OJ, Gelabert A, Rosales A, Coronado J. Femoral neuropathy after transuretral endoscopic surgery: a rare complication. Actas Urol Esp 1987;11(5):489-90.

24. Katiriji MB, Lanska DJ. Femoral neuropathy after radical prostatectomy. Urology 1990; 36(6): 539-540.

25. Ujike T, Meguro N, Tanikawa G, Kakimoto K, Ono Y, Maeda O, Kinouchi T, Usami M. Two cases of femoral nerve palsy alter radical prostatectomy. Hinyokika Kiyo 2005; 51(4):297-299.

26. Teruel JL, Martín Escobar E, Marcen R, Berenguer A, Ortuño J. Neuropatía como complicación quirúrgica del transplante renal. Actas Urol Esp 1984; 8(2):137-140.

27. Jost L (H), Jost L, Nogues M, Davalos M, Turin M, Manes F, Leiguarda R. Complicaciones neurológicas del transplante renal. Medicina (B Aires). 2000; 60(2): 161-164.

28. Vaziri ND, Barton CH, Ravicumar GP, Martin DC, Ness R, Siki J. Femoral neuropathy: a complication of renal transplantation. Nephron 1981;28:30-31.

29. Yazbeck S, Larbrisseau A, O’Regan S. Femoral neuropathy after renal transplantation. J Urol 1985;134:720-721.

30. Monga M, Castañeda Zuñiga WR, Thomas R. Femoral neuropathy following percutaneous nefhrolithotomy of a pelvic kidney. Urology 1995; 45(6): 1059-1061.

31. Ramon S, Nativ O, Apter S,Goldwaser B. Renal cell carcinoma presenting as femoral neuropathy. Isr $\mathrm{J}$ Med Sci 1989; 25(4):233-234.

32. Grenier G, Aboulker P. La paralysie crurale. Complication de la chirurgie de l'uretere pelvien. Ann Chir 1967; 21(9): 593-596.

33. Kowalczyk ER, Keating MA, Ehrlich RM. Femoral nerve neuropathy after the psoas hitch procedure. Urology 1996; 47(4):563-565.

34. Schneck SA. Perifheral and cranial nerve injuries resulting from general surgical procedures. Arch Surg 1960;81:855859.

35. Kabalin JM. Femoral neuropathy following abdominal operations for genitourinary malignancies. Urology 1995;45: 1089.

Correspondencia autor: Dr. J.Mª Pastor Guzmán

Servicio de Urología. Hospital General Universitario de Albacete Hermanos Falcó, s/n - 02006 Albacete

Tel.: 967597100

E-mail autor: jmpastorg@hotmail.com Información artículo: Urología general

Trabajo recibido: diciembre 2006

Trabajo aceptado: abril 2007 